\title{
Assessment of genome annotation using gene function similarity within the gene neighborhood
}

\author{
Se-Ran Jun ${ }^{1 *}$ (D), Intawat Nookaew ${ }^{1}$, Loren Hauser ${ }^{2}$ and Andrey Gorin ${ }^{3}$
}

\begin{abstract}
Background: Functional annotation of bacterial genomes is an obligatory and crucially important step of information processing from the genome sequences into cellular mechanisms. However, there is a lack of computational methods to evaluate the quality of functional assignments.

Results: We developed a genome-scale model that assigns Bayesian probability to each gene utilizing a known property of functional similarity between neighboring genes in bacteria.

Conclusions: Our model clearly distinguished true annotation from random annotation with Bayesian annotation probability $>0.95$. Our model will provide a useful guide to quantitatively evaluate functional annotation methods and to detect gene sets with reliable annotations.
\end{abstract}

Keywords: Genome functional annotation, Gene function similarity, Gene neighborhood, Bayesian probability

\section{Background}

During recent years, technological advances have enabled the rapid and affordable sequencing of organisms from all kingdoms of life. In 2011 the volume of the NCBI Sequence Read Archive crossed a remarkable size of $100 \mathrm{~TB}$ [1], and more than 22,000 complete or nearly complete genomes are available for bacterial organisms with the number increasing by $>1000$ each month $[2,3]$. Functional annotation of bacterial genomes is an obligatory and crucially important step of information processing from the genome sequences toward insights into cellular mechanisms, putative ecological roles, or predictive models of a given organism or microbial community. Numerous software packages, databases, platforms, and score filters involve computational pipelines that assign functions to the genes [4]. However, the sequence information is only as good and useful as the functional annotation when it has functional annotation attached to it. The function of genes is central for all biological insights, including interpretation and design of experiments and comparative genomic analysis, as well as the

\footnotetext{
* Correspondence: sjun@uams.edu

${ }^{1}$ Department of Biomedical Informatics, College of Medicine, University of

Arkansas for Medical Sciences, Little Rock, AR 72205, USA

Full list of author information is available at the end of the article
}

input data for metabolic and regulatory models $[5,6]$. The manual curation or experimental verification [7] is unlikely to be feasible when $>1000$ genomes are added each month. Accordingly, there is a greater urgency to have computational tools for genome annotation validation [8].

In the literature, "annotation quality" sometimes refers to the precision of finding an exact start site for the genes in the genome $[8,9]$. When the location of a gene is determined incorrectly, it follows that functional annotation will more likely be incorrect as well. Therefore, the gene finding problem is an important part of the process for genome annotation. In this work, we aim to address annotation consistency at the level where genes are found and annotated by standard protein function annotation, Gene Ontology (GO) terms, organized in a hierarchical fashion [10]. The benefits of function annotation by $\mathrm{GO}$ are a systematic control vocabulary that enables cross-comparison over different genomes and a higher percentage of genes in the genome that can be annotated because of different levels of information of GO hierarchy.

In an approach described by Skunca et al. [11], the authors measured the annotation quality of individual GO terms using experimental verifications and estimated the annotation quality of the database UniProt-GOA 
over time. This approach dealt with relatively small datasets composed of model organisms because it was dependent on experimental verifications. Alternatively, the occurrence of annotation terms was used in a recent computational study [12], which indicated that the manually curated annotations have more natural lexical properties than automatically generated ones, but this method was a bulk analysis within the annotation database and it does not describe the annotation quality of any particular genome. In other studies, authors have used multiple tools and performed manual analysis of the problematic annotations $[13,14]$. These are reliable approaches, but they are clearly not scalable to dozens of genomes.

Our approach to the validation of gene annotation utilized a well-known and fundamental property of the bacterial genomes: functionally coordinated genes tend to be physically closer on a chromosome than the average gene [15-17]. However, this property was rarely used by others except in a semiquantitative way [18], which used the property to find functional annotations especially for difficult cases of hypothetical proteins. The novel idea of our work (described in Methods in detail) is illustrated in Fig. 1. In this study, a gene neighborhood is defined as three left and right genes of a given gene along the chromosome. We developed an analytical approach to measure gene function similarity (GFS) for each neighboring pair of genes, applied Bayesian statistics to integrate gene neighborhood information of annotation, and then finally, computed the probability of annotation confidence (PAC) for each gene that has at least one GFS score available within its neighborhood, given that functional assignment with very few and well-controlled empirical assumptions is correct. Our method provides genome annotation assessment through the annotation evaluation of all individual genes in the genome.

\section{Results}

Probability of annotation confidence

We applied our methodology to Escherichia coli and Clostridium thermocellum to calculate the PAC for NCBI annotation (assumed to be a well annotation) and compared it with "random" annotation. For each gene with an annotation in E. coli, the random annotation was generated by assigning a random annotation selected from 8

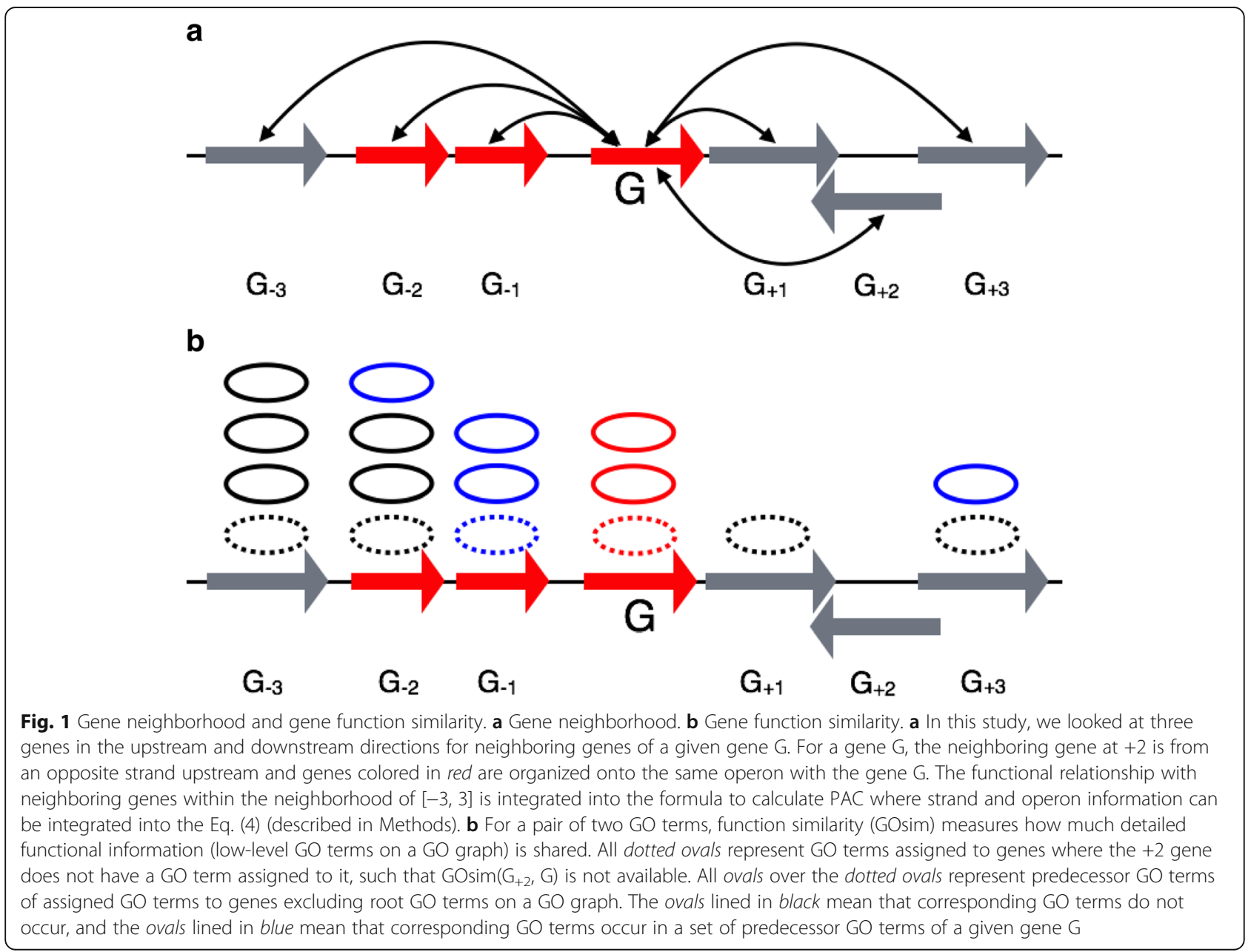


million bacterial and archael proteins from UniProtKB/ Swiss and UniProtKB/TreEMBL [19] and the NCBI Reference Sequence databases [20]. Note that the random annotation may happen to be correct or partially correct by chance. Figure 2a shows histograms of PAC values (which are Bayesian annotation probabilities described in Methods) for E. coli and Fig. 2b for C. thermocellum for $\mathrm{NCBI}$ annotations and simulated random annotations. For the study in Fig. 2, the simplest model was considered where the independence of function similarities within the gene neighborhood was assumed and information for the operon and strand was not integrated. Note that conditional probabilities derived from each genome were applied to the genome, respectively, for the PAC calculations in Fig. 2. The total number of genes considered in Fig. 2a was 3117 (of 4147 genes), among which 1021 genes had a probability range from 0.95 to 1.00. The distribution of probabilities of the random annotations showed only 49 genes in the probability bin $[0.95,1]$. The NCBI annotations with lower PAC values may come from an insufficient number of detectable function similarities with genes in the neighborhood that were derived from the uncovered knowledge of GO annotation and graph structure. We proposed to use a fraction of genes in the probability bin $[0.95,1]$ as the annotation quality score (AQS) showing distinct differences between NCBI annotation and random annotation. Hence, the NCBI annotation

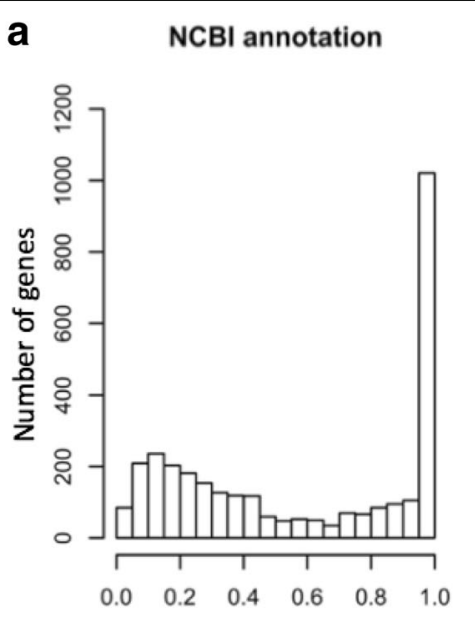

Probability of annotation confidence

b

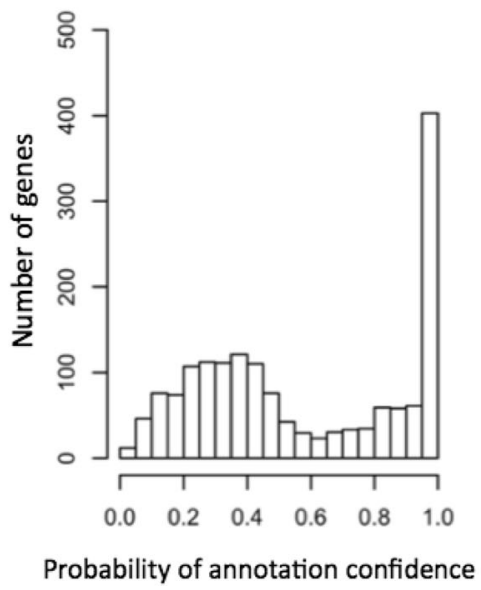

Random annotation

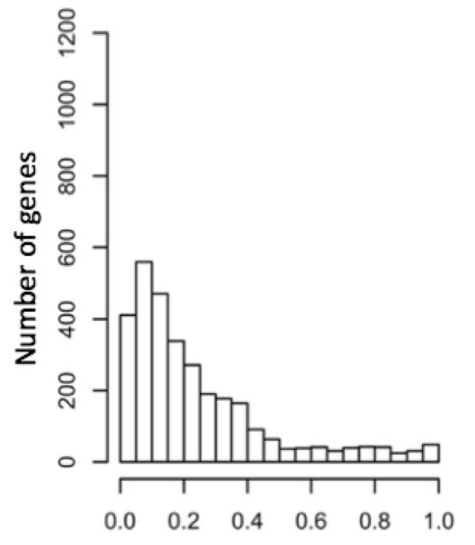

Probability of annotation confidence

Random annotation

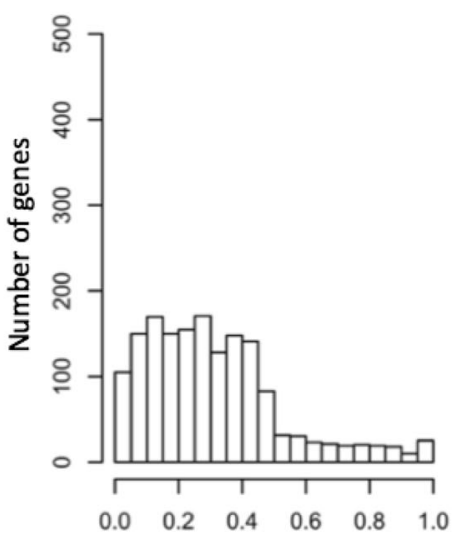

Probability of annotation confidence

Fig. 2 Distributions of PAC values of NCBI and random annotations for (a) E. coli and (b) C. thermocellum. a Using conditional probabilities derived from a given genome and observed gene function similarities, we calculated PAC values for NCBI annotation (assumed to be correct) and random annotation (assumed to be incorrect) for the E. coli strain K-12 substrain MG1655. The probability bin [0.95, 1] has 1021 genes for $\mathrm{NCBI}$ annotation and 49 genes for random annotation of 3117 genes applicable to PAC calculation. $\mathbf{b}$ We applied the same methodology to C. thermocellum. The probability bin $[0.95,1]$ contains 403 genes for NCBI annotation and 25 genes for random annotation among 1617 genes applicable to PAC calculation 
of $E$. coli has an AQS of $0.33(=1021 / 3117)$ and the random annotation of $E$. coli has an AQS of 0.016 $(=49 / 3117)$. The analogous distributions to C. thermocellum were plotted in Fig. $2 \mathrm{~b}$, and the AQS for C. thermocellum NCBI annotation amounted to 0.24, whereas its random annotation had a similar score to E. coli, 0.015. We used C. thermocellum as an example of a genome that is evolutionarily distant from E. coli and most certainly is more difficult to annotate as comprehensively as $E$. coli. The $C$. thermocellum annotation contained a large number of hypothetical genes ( $\sim 31 \%$ of the genome), as well as genes with annotations not fitted into GO classification ( 16\%). As a result of those adverse factors, only 1617 genes were applicable to a PAC calculation, such that it is reasonable for the AQS for $C$. thermocellum to be lower than the one for E. coli, but the difference is not overwhelmingly huge. Figure 3 provides another important assessment for checking the developed methodology. Figure $3 \mathrm{a}$ and $\mathrm{b}$ accumulated all collected annotations (correct plus incorrect annotation) for each probability bin. The $x$-axis represents the right-end PAC value (Bayesian annotation probability) for a bin and the $y$-axis represents the fraction of true annotations among annotations collected for the bin. On both plots, our model showed a slight overestimation (points over diagonal) and underestimation (points under diagonal) of the sensitivity. However, the probability bin $[0.95,1]$ showed sensitivity fairly close to the diagonal. Furthermore, both diagonal plots looked almost identical, suggesting the robust properties of the developed methodology even though the annotation of $C$. thermocellum showed sparse functional annotation compared to $E$. coli.

\section{Operon structure inclusion into the PAC}

So far, we have shown results generated from the simplest model, which used gene function similarities within the gene neighborhood that are assumed to be independent of each other, and clearly distinguished a good quality of annotation from random annotation with the PAC. Yet, a simple integration of the operon structure, which would introduce a separate uncertainty factor in the analysis, could be done by a hybrid system that uses operon-derived conditional probabilities for the genes that are certainly in the same operons and another set of probabilities for the genes that are not. However, in this study, we explored operon structure into PAC by counting only the neighboring genes that are deemed to be on the same operon with a given gene in the formula (4) in Methods. For E. coli, inclusion of the operon structure showed rather dramatic changes in the distribution of PAC values in Fig. 4. First, the number of genes with assigned probabilities was reduced significantly because pairs of genes on the same operon were only considered when calculating gene function similarity. The probabilities were assigned only to 1816 genes of 3117 genes in the "no-operon" model. However, there were still 916 genes found in the highly reliable category $[0.95,1] \mathrm{com}$ pared to 1021 for the no-operon model (50\% of genes for the operon model versus $33 \%$ of genes for the nooperon model in the bin $[0.95,1])$. The distribution of PAC values in Fig. 4 was much cleaner in a sense that a lower number of genes with PAC values $<0.95$ were found but still showed a similar shift. However, the distribution for the random annotations had a peak around 0 probability. Summarizing the statement above, Fig. 5 represents the normalized number of genes with PAC

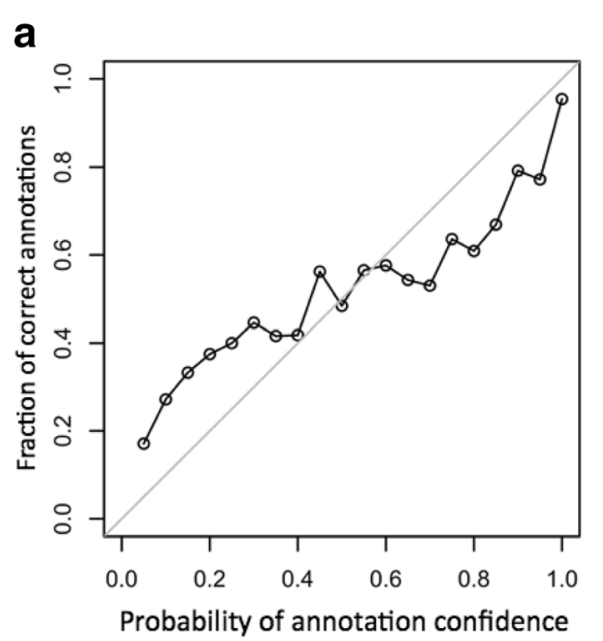

b

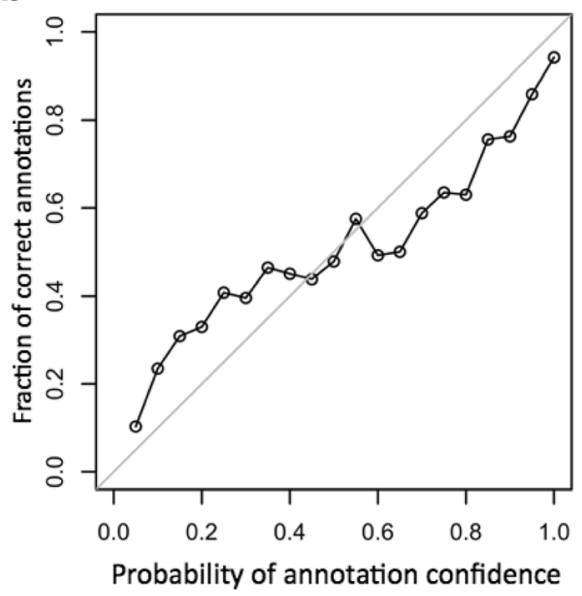

Fig. 3 Diagonal plots of fractions of correct annotations for (a) E. coli and (b) C. thermocellum. The $x$-axis represents the right-end PAC value for a given bin, and the $y$-axis represents a fraction of correct annotations (NCBI annotations) among all annotations (correct and incorrect) collected for the bin. The points over and under the diagonal indicate overestimation and underestimation of fractions of correct annotations, respectively. In general, we observed points fairly close to the diagonal with both plots 


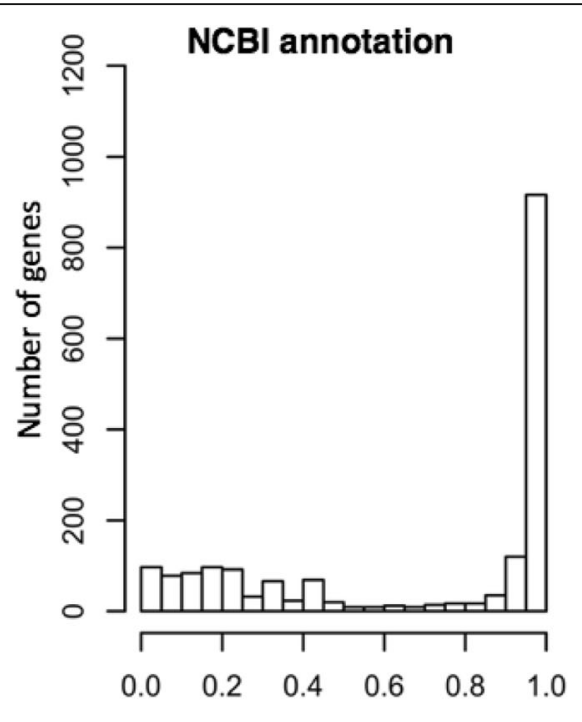

Probability of annotation confidence

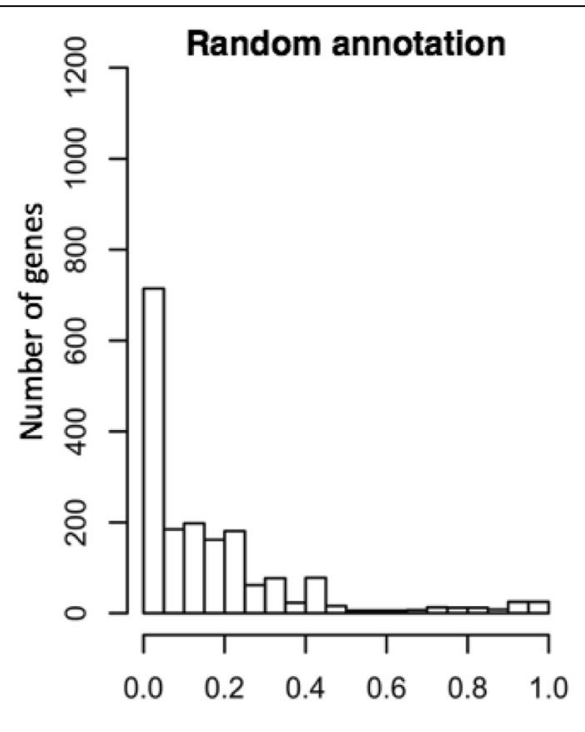

Probability of annotation confidence

Fig. 4 Operon structure inclusion into annotation probability with E. coli. The predicted operon information of E. coli was integrated in PAC values by considering genes on the same operons for NCBI and random annotation

values by the total number of genes applicable to PAC calculation for the no-operon and operon models, respectively. Both plots clearly showed that inclusion of the operon structure into our model contributes to a better distinction between NCBI annotation and random annotation.

\section{Experiments with gene shuffling}

To investigate how our model for annotation validation responds to the increased number of incorrect annotations, we generated annotations with "almost correct functional predictions" through "disturbances by gene shuffling" with NCBI annotation of $E$. coli. In each experiment, we randomly selected $\mathrm{Nr}$ pairs of genes with annotations by GO terms and exchanged annotations of the selected pairs where annotations were only used once for shuffling. The shuffling procedure was repeated 100 times for each $N r$. Figure 6 represents distributions of PAC values of the shuffled annotations where each column shows the average number of genes within a probability bin over 100 repeats and the error bars show 1 standard deviation (SD). Figure 6a was constructed for $N r=100$, such that 200 genes likely had the wrong annotations. We did not make any additional check on the shuffling process to determine whether it is possible that the shuffling process would swap close or even identical annotations. The SD was small for all probability bins. For example, the average and SD for the probability bin
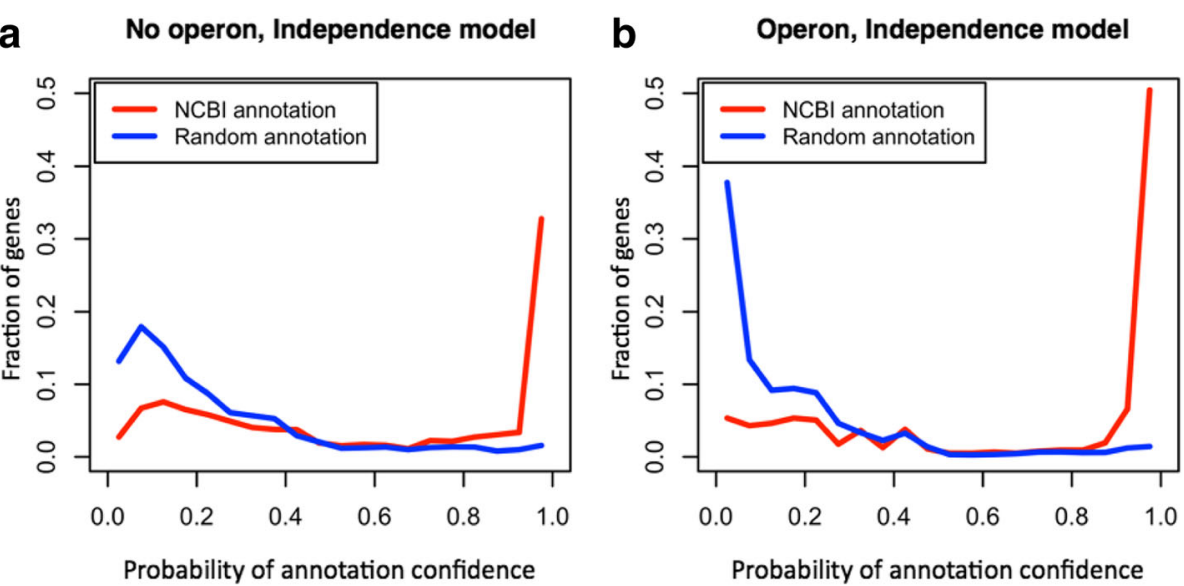

Fig. 5 Comparison of no-operon and operon models with $E$. coli. The $y$-axis represents the normalized number of genes within a probability bin by the total number of genes applicable for PAC calculation (a) without and (b) with operon structure inclusion 

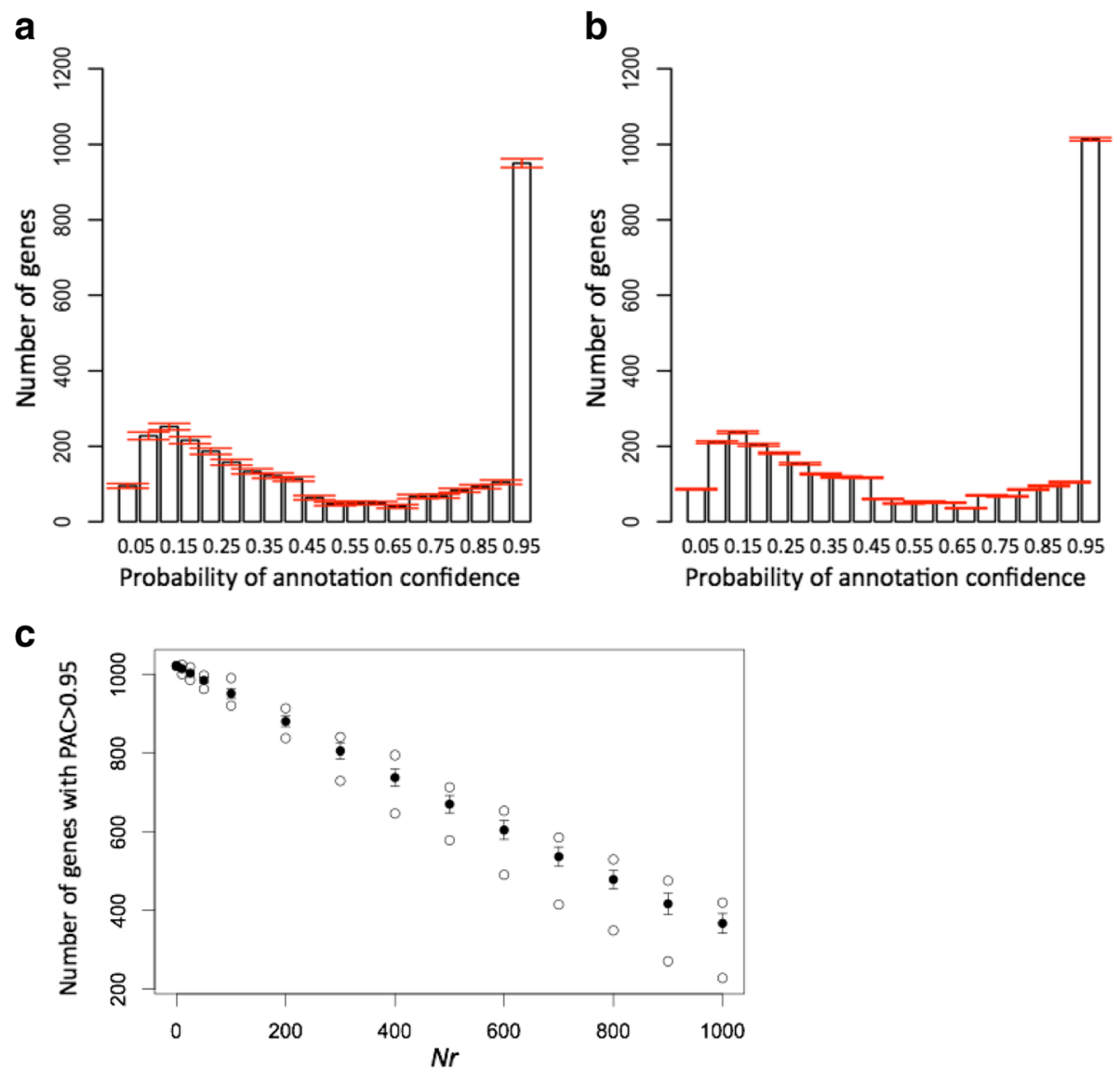

Fig. 6 Gene shuffling experiments with E. coli. a Shuffle for $N r=100$. b Shuffle for $N r=10$. c Shuffle summary. $\mathbf{a}$ and $\mathbf{b}$ The distributions of PAC values were plotted for the shuffled assignments with E. coli. In each experiment, the Nr pairs of genes with annotations by GO terms were randomly selected and gene annotations in each pair were exchanged. For each Nr, the experiment was repeated 100 times, and the plots represent the average number of genes with the SD observed for each probability bin. c The average (black dot), SD (vertical lines), and maximum and minimum (white dot) number of genes were presented for the probability bin [0.95, 1.00] for $\mathrm{Nr}=10,25,50,100,200$, and up to 1000 (shown on the $x$-axis)

$[0.95,1]$ were 950.5 and 11.6 , respectively, which it is about $6 \mathrm{SD}$ away from the value observed for canonical annotation (1021 genes). In Fig. 6b, we observed that our model remains very sensitive to the annotation disturbance of only 20 genes $(\mathrm{Nr}=10)$ for the $E$. coli genome composed of $>4000$ genes. We had 1013.9 on average with 4.4 SD in the bin $[0.95,1]$, which is still $\sim 2 \mathrm{SD}$ away from the undisturbed annotation (1021 genes). In Fig. 6c, the average (black dot), SD (vertical line), and maximum and minimum (white dot) number of genes for the probability bin $[0.95,1.00]$ were presented for $N r=10,25,50,100$, 200 , and up to 1000 (shown on the $x$-axis). Overall, a linear dependency between the number of shuffling, $\mathrm{Nr}$, and a decrease in the (average) number of the genes with highly reliable annotations was observed.

\section{Discussion}

Here we discuss possible enhancements and further developments with potential gains in the model performance:
(1) one could explore distance to define neighboring genes as a parameter. For example, one can use basepairs of physical distance along the chromosome as a threshold to define gene neighbors instead of 3 genes upstream and downstream, which is currently used. (2) We treated all genes equally in the current experiments, but in reality the annotations of some genes would be absolutely certain. It would not be difficult to include into our system as another category of genes, "annotation anchors", and then compute a separate set of conditional probabilities of gene function similarities for such genes. (3) We appended another gene neighborhood structure, "strand information", into the Bayesian formula with $E$. coli for which we derived conditional probabilities for a set of genes on the same strand and another set of genes not on the same strand. In the Additional file 1: Figure S1 represents PAC distributions calculated from strand-integrated conditional probabilities for NCBI and random annotations, which showed a slightly better performance than those obtained 
from the model without strand information, in a sense that 1042 genes were found in the bin $[0.95,1]$ for NCBI annotation, whereas 42 genes for random annotation were found in the bin $[0.95,1]$. (4) For all results shown, we extracted the conditional probabilities from Eq. (4) in Methods (likelihood in Bayes' rule) derived from a given genome. However, $C$. thermocellum was not annotated by functional terms as much as $E$. coli comprehensively, which led to a much lower number of gene pairs with functional annotations, that might not produce enough data to estimate conditional probabilities (likelihood in Bayes' formula) for probabilistic modeling. To further evaluate robustness toward conditional probabilities, we applied conditional probabilities derived from $E$. coli to calculate the PAC of genes in C. thermocellum for NCBI annotation and random annotation. We observed distributions of the PAC values obtained with conditional probabilities derived from $E$. coli similar to those obtained with conditional probabilities derived from $C$. thermocellum in Additional file 1: Figure S2. In the future, we plan to specifically explore this question for a large number of bacterial genomes, yet the result with $C$. thermocellum was very encouraging, even though it is evolutionarily rather distant from E. coli. (5) We explored the COG database [21] to annotate genes by functional terms and generated PAC values. Ignoring a poorly characterized functional category, the COG functional terms are organized into three hierarchical levels where the first level consists of three functional classes (Information Storage and Processing, Cellular Processes and Signaling, Metabolism), the finer sub-functional classes (23 functional classes at the second level), and COG terms at the third level. Note that some COG terms belong to more than one functional class. To generate random COG annotation for each protein with an assigned COG term, we assigned a COG term for a protein randomly chosen within the genome to the given protein. The conditional probability of an observation profile given correct and incorrect annotation was calculated for each functional category at the first level where gene COG function similarity takes two values: 0 if two genes share a COG term, and 1 otherwise. In Additional file 1: Figure S3, which represents PAC distributions for NCBI annotation and random annotation with $E$. coli, we obtained an AQS of 0.17 (419/2498 where 2498 proteins were applicable to PAC calculation) for NCBI annotations and an AQS of 0.04 (95/2498) for random annotations that $\mathrm{COG}$ annotation showed a less obvious distinction between NCBI and random annotation than $\mathrm{GO}$ annotation in the probability bin $[0.95,1]$. In the future, we will explore other functional annotation databases including KEGG Orthology [22] and PFAM [23] and compare corresponding PAC distributions for genome annotation validation. (6) So far, we discussed experiments under the "independent" Bayesian model. For example, we approximated the conditional probability of GFSs in the neighborhood as a product of conditional probabilities of individual GFSs within the gene neighborhood. To investigate the influence of the assumption of independence on the AQS, we formulated Bayesian annotation probability under the dependent model, which is described in detail in the Additional files 1 and 2. For the dependent model, we assumed that observations made downstream and upstream depend on only a given gene, and an observation $\mathrm{O}_{i}$ depends on an observation $\mathrm{O}_{\mathrm{i}+1}$ in the downstream and $\mathrm{O}_{\mathrm{i}-1}$ in the upstream. The distributions of PAC values under the dependent model for $E$. coli are presented in Additional file 1: Figure S4. Under the dependent model considered in this study, we did not observe any gain in terms of the AQS, which is probably due to the assumption not fitting the biological expectation and not enough data to reliably estimate dependency. The main incentive to use it, in any case, is to avoid overestimation and underestimation of PAC calculation, which was not a problem as shown in Fig. 3.

Currently, we envision three possible application directions for the proposed genome-scale model. First, when the different annotation pipelines annotate the same bacterial genomes, our model should be able to compute a measure of consistency for each annotation pipeline; i.e., AQS, the fraction of the genes with a PAC value $>0.95$. The workflow with a better score would likely have more correct assignments because our genome-scale probabilistic model sensitively captures the small difference in annotations as shown in the Experiments with gene shuffling section. For example, we compared two C. thermocellum genomes annotated at different times where one (called old annotation) was annotated on Feb 14, 2007 at GenBank, and the other genome downloaded from NCBI on May 2013 (called new annotation) was used in this study. The old annotation had 1658 proteins (of 3198 total proteins) annotated with GO terms among which 1582 proteins were applicable to PAC calculation, which resulted in 349 proteins in the bin $[0.95,1]$ leading to an AQS of $0.22(=349 / 1582)$. The new annotation had 1671 proteins (of 3173 total proteins) annotated with GO terms applicable to PAC calculation, which resulted in 403 proteins in the bin $[0.95,1]$ leading to an AQS of 0.24 (= 403/1671). The comparison of $C$. thermocellum genomes annotated at different times may support that our model could be a quantitative tool for genome annotation validation. Second, we plan to measure the annotation consistency for many different bacteria (possibly for 32,000 genomes stored by Land et al. [3]), and such research should provide reasonable estimates of which values are reliable for various branches of the tree of life. Finally, individual PAC values should be valuable for the evaluation of hypothetical protein annotation unless functional inference of hypothetical 
proteins does not exploit gene neighborhood information as happened in other studies [17, 24].

\section{Conclusions}

Sequencing technologies continue to develop rapidly, and the list of genes with assigned functions is the main product of the sequencing efforts, as it is used to further research. However, there is a lack of methods to evaluate the quality of the obtained functional assignments. We developed a genome-scale probabilistic model that quantitatively measures annotation consistency relying on the well-established property of bacterial genomes; i.e., genes lying in physical adjacency on a chromosome tend to be associated functionally. To our knowledge, this is the first tool that provides both a quality value for the whole set of genes as well as probability of the annotation confidence for individual genes in the set. We have tested our method by simulating large and small "disturbances" of the functional assignments, and the method proved to be sensitive for both cases. The range of potential applications is wide including evaluation and comparison of standard annotation methods for functional assignment. This will lead to more biological insights and more precise cellular models as both use functional assignments as input information.

\section{Methods}

\section{Data}

In this study, the genome-scale probabilistic model was first applied to assess the annotation of two genomes: $E$. coli str. K-12 substrain MG1655 (NC_000913.faa) and C. thermocellum ATCC 27405 (NC_009012.faa) downloaded from NCBI. The background comparison by random annotation of a genome was performed by randomly picking a protein annotated by functional terms from the protein sequence database. The protein sequence database for random assignments was downloaded from the UniProtKB/Swiss, UniProtKB/TreEMBL [19], and NCBI Reference Sequence [20] databases, which included 8 million bacterial and archeal proteins. The most current version of the same dataset is at least five times as large, but this factor is not important for our particular study.

\section{GO for functional annotation}

To quantitatively assess the annotations, we translated annotations using a controlled vocabulary system, the GO project [10]. The approach to use GO for an evaluation of gene function similarities has been used previously [11, 25], but to our knowledge it has not been used for comprehensive evaluation of genome annotation quality. The GO project describes the ontology of defined GO terms representing gene product properties structured as a directed acyclic graph. The directed graph can be retrieved from "gene_ontology.1_2.obo.txt"
[26] which contains GO terms annotated by both the experimental and computational evidence codes. The directed GO graph covers biological process, molecular function, and cellular component, which are mutually exclusive domains each represented by the root GO terms separately. The directed relationships between GO terms represent either "is-a", "part of", or "regulates" where child terms are more specialized and parent terms are less specialized. Some GO terms may have more than one parent term unlike a hierarchy. In this work, we considered directed edges, which represent only the "is-a" subclass relationship. The UniProt Gene Ontology Annotation (UniProt-GOA) database provides highquality GO annotations to proteins through the UniProt Knowledgebase. To annotate NCBI annotations by GO terms, we first assigned NCBI GI numbers to the UniprotKB identifier using "idmapping.dat" [26], and then assigned a UniprotKB identifier into GO terms using "gene_association.goa_uniprot" [27]. Note that the mapping between NCBI GI numbers and UniprotKB identifiers is not one-to-one, and some NCBI GI numbers are not mapped into a UniprotKB identifier.

\section{Gene function similarity}

We introduced GO similarity to compare quantitatively functional annotations described by GO terms. To calculate functional similarity between two $\mathrm{GO}$ terms $\left(\mathrm{GO}_{1}\right.$, $\mathrm{GO}_{2}$ ), we first identified a set of all predecessor GO terms of $\mathrm{GO}_{1}\left(\mathrm{GO}_{2}\right)$ on the directed $\mathrm{GO}$ graph including $\mathrm{GO}_{1}$ $\left(\mathrm{GO}_{2}\right)$ but excluding the root, denoted by $\mathrm{S}_{1}\left(\mathrm{~S}_{2}\right)$, respectively. Then, the similarity between two GO terms was defined based on overlapping GO terms between sets $S_{1}$ and $S_{2}$ as follows:

$$
\operatorname{GOsim}\left(G O_{1}, G O_{2}\right)=\frac{\left|S_{1} \cap S_{2}\right|}{\left|S_{1} \cup S_{2}\right|}
$$

where $\left|S_{1} \cap S_{2}\right|$ and $\left|S_{1} \cup S_{2}\right|$ are the cardinalities of an intersection and the union of $S_{1}$ and $S_{2}$, respectively. The normalized GO similarity, which falls in the range of 0 to 1 , implicitly measures more than just the detailed functions (low-level GO terms) that are shared. For instance, in Fig. 1b, all dotted ovals represent GO terms assigned to genes where the +2 gene does not have a GO term assigned to it, such that $\operatorname{GOsim}\left(G_{+2}, G\right)$ is not available. All ovals over the dotted ovals represent predecessor GO terms of assigned GO terms to genes excluding the root GO term on a directed GO graph. The ovals lined in black mean that corresponding GO terms do not occur, and the ovals lined in blue mean that corresponding GO terms occur in a set of predecessor GO terms of a gene G. Therefore, GO similarities between neighboring genes and gene $G$ are as follows: $\operatorname{GOsim}\left(G_{-3}\right.$, G) $=0, \operatorname{GOsim}\left(G_{-2}, G\right)=1 / 6, G \operatorname{Osim}\left(G_{-1}, G\right)=1$, 
$\operatorname{GOsim}\left(G_{+1}, G\right)=0, G \operatorname{Osim}\left(G_{+3}, G\right)=1 / 4$. However, genes can be annotated with more than one GO term because proteins can have multiple functional roles. Let's say that gene $G_{1}$ is annotated with $A_{1}=\left\{G_{i} \mid i=1, . ., M\right\}$ and gene $\mathrm{G}_{2}$ with $\mathrm{A}_{2}=\left\{\mathrm{GO}_{\mathrm{j}} \mid j=1, . ., \mathrm{N}\right\}$, the GFS between genes $G_{1}$ and $G_{2}$ is defined as the maximum among $G O$ similarities between two GO terms from different genes:

$$
\operatorname{GFS}\left(G_{1}, G_{2}\right)=\max _{\substack{1 \leq i \leq M \\ 1 \leq j \leq N}} \operatorname{GOsim}\left(G O_{i}, G O_{j}\right)
$$

where $G_{i}$ is from gene $G_{1}$ and $G_{j}$ is from gene $G_{2}$. The maximum of GO similarities takes into account different numbers of GO terms assigned to different proteins. We calculated the GFS associated with each biological process, molecular function, and cellular component separately.

\section{Gene neighborhood structure}

In this study, we explored three different gene neighborhood structures: gene order on a chromosome, operon structure, and strand information. The strand information of genes was retrieved through the NCBI Entrez Programming Utilities. For the predicted operon structure of E. coli, we used the Database of Prokaryotic Operons [28]. For each gene $\mathrm{G}$ and each functional category (biological process, molecular function, and cellular component) in a given genome, we calculated $\operatorname{GFS}\left(G, G_{i}\right)$ between $G$ and its neighbor gene $G_{i}$ at $i$ th neighborhood, $i=-3,-2,-1,+1,+2,+3$, where the minus and plus signs represent upstream and downstream neighborhoods (Fig. 1a).

\section{Deriving PAC through Bayes' rule}

Here we derived the probability that annotation of a gene $\mathrm{G}$ is correct in given observations $\left\{\mathrm{O}_{\mathrm{i}} \mid i=-3, \ldots,+3\right\}$ with neighbor genes $\mathrm{G}_{\mathrm{i}}, i=-3, \ldots,+3$ (called an observation profile), under the assumption that observations are independent of each other within the gene neighborhood. First, we calculated conditional probability (likelihood in Bayes' rule) that an observation $\mathrm{O}_{\mathrm{i}}$ is observed at the $i$ th neighborhood given the correct annotation, denoted by $\operatorname{Pr}\left(\mathrm{O}_{\mathrm{i}} \mid \mathrm{A}_{\mathrm{c}}\right)$, where $\mathrm{A}_{\mathrm{c}}$ represents correct annotation, for which NCBI annotation and corresponding functional annotation by GO terms were all assumed to be correct. Then, we calculated the probability that an observation $\mathrm{O}_{\mathrm{i}}$ is observed at the $i$ th neighborhood given the incorrect annotation, denoted by $\operatorname{Pr}\left(\mathrm{O}_{\mathrm{i}} \mid \mathrm{A}_{\text {inc }}\right)$, where $A_{\text {inc }}$ represents incorrect annotation, for which we generated an annotation for each protein with assigned GO terms by randomly drawing a protein with assigned GO terms from the database of 8 million proteins, and then assigning the GO terms of the randomly drawn protein to the given protein. For each protein, we calculated gene function similarity with gene neighbors using the given gene's random annotation, leading to $\operatorname{Pr}\left(\mathrm{O}_{\mathrm{i}} \mid \mathrm{A}_{\text {inc }}\right)$. If we formulate conditional probabilities using gene function similarity, then a random variable $\mathrm{O}_{\mathrm{i}}$ takes $\mathrm{GFS}_{\mathrm{i}}$, where $\mathrm{GFS}_{\mathrm{i}}$ represents gene function similarity between genes separated by $(i-1)$ genes on a chromosome. The use of combinatorial information of gene neighborhood structures can be easily integrated into the formula. Based on Bayes' rule along with the assumption of independence of neighbor observations, the probability that an annotation is correct given an observation profile is described as follows:

$$
\begin{aligned}
\operatorname{Pr}\left(A_{c}, \mid O_{i}, i=-3, \cdots,+3\right) & \frac{\operatorname{Pr}\left(O_{i}, i=-3, \cdots,+3, \mid A_{c}\right) \operatorname{Pr}\left(A_{c}\right)}{\operatorname{Pr}\left(O_{i}, i=-3, \cdots,+3, \mid A_{c}\right) \operatorname{Pr}\left(A_{c}\right)+\operatorname{Pr}\left(O_{i}, i=-3, \cdots,+3, \mid A_{\text {inc }}\right) \operatorname{Pr}\left(A_{\text {inc }}\right)} \\
= & \frac{\prod_{i=-3}^{i=+3} \operatorname{Pr}\left(O_{i}, \mid A_{c}\right) \operatorname{Pr}\left(A_{c}\right)}{\prod_{i=-3}^{i=+3} \operatorname{Pr}\left(O_{i}, \mid A_{c}\right) \operatorname{Pr}\left(A_{c}\right)+\prod_{i=-3}^{i=+3} \operatorname{Pr}\left(O_{i}, \mid A_{\text {inc }}\right) \operatorname{Pr}\left(A_{\text {inc }}\right)},
\end{aligned}
$$

where $\operatorname{Pr}\left(\mathrm{A}_{\mathrm{c}}\right)$ and $\operatorname{Pr}\left(\mathrm{A}_{\text {inc }}\right)$ are prior probabilities of correct and incorrect annotations respectively, which were set to 0.5 in this study. By considering all three functional categories concurrently, the Bayesian annotation probability (called the PAC in this study) is described as follows:

$$
\begin{aligned}
\operatorname{Pr}\left(A_{c} \mid O_{i}^{B P}, O_{i}^{M F}, O_{i}^{C C}, i=-3, \cdots,+3\right) \\
=\frac{\operatorname{Pr}\left(O_{i}^{B P}, O_{i}^{M F}, O_{i}^{C C}, i=-3, \cdots,+3 \mid A_{c}\right) \operatorname{Pr}\left(A_{c}\right)}{\operatorname{Pr}\left(O_{i}^{B P}, O_{i}^{M F}, O_{i}^{C C}, i=-3, \cdots,+3\right)} \\
=\frac{\prod_{i=-3 j}^{i=+3} \prod_{i=B P}^{C C} \operatorname{Pr}\left(O_{i}^{j} \mid A_{c}\right) \operatorname{Pr}\left(A_{c}\right)}{\prod_{i=-3 j=B P}^{i=+3} \prod_{i=+3}^{C C} \operatorname{Pr}\left(O_{i}^{j} \mid A_{c}\right) \operatorname{Pr}\left(A_{c}\right)+\prod_{i=-3 j=B P}^{C C} \operatorname{Pr}\left(O_{i}^{j} \mid A_{\text {inc }}\right) \operatorname{Pr}\left(A_{\text {inc }}\right)}
\end{aligned}
$$

where BP indicates biological process; MF, molecular function; and $\mathrm{CC}$, cellular component. For example, if a random variable $\mathrm{O}_{\mathrm{i}}$ takes a two-dimensional vector of gene function similarity and strand information for each category, then Bayesian annotation probability in the formula (1) is derived from an 18-dimensional observation vector. In most cases, we do not have all neighbor genes with assigned GO terms for all categories. The nonexistent information elements are silently ignored in the formula (4) under the assumption that non-existent information occurs equally in correct annotation and incorrect annotation.

\section{Filtering abundant $\mathrm{GO}$ terms}

The GFS is affected by GO terms with an abundant occurrence due to their general functional description; 
for example, GO:0016020, which describes a membrane in a category of the cellular component. Therefore, the GO terms with high frequency can cause random pairs of genes that are not neighbors on a chromosome to share functions, eventually yielding high Bayesian annotation probability. In the Additional file 1; Figure S5 represents the frequency of $\mathrm{GO}$ terms in a percentage of proteins with assigned $\mathrm{GO}$ terms in the protein sequence database. To avoid false causality with Bayesian annotation probability, we filtered out GO terms whose frequencies were $>5 \%$. For 10,000 random protein pairs with assigned GO terms in the protein sequence database, Additional file 1: Figure S6A represents histograms of GFS values before filtering abundant GO terms and Additional file 1: Figure S6B shows GFS values after filtering abundant $\mathrm{GO}$ terms with a frequency $>5 \%$ in each functional category. In the Additional file 1: Table S1 lists GO terms that were filtered out with a functional description and a $5 \%$ of frequency cutoff. All results shown in our study were derived after filtering GO terms with a $5 \%$ of frequency cutoff.

\section{Additional files}

Additional file 1: supplementary.doc. Supplementary Figures and Tables. (DOC 947 kb)

Additional file 2: Cthermocellum_oldannotation.txt. The old annotation of C. thermocellum. (TXT $364 \mathrm{~kb}$ )

\section{Abbreviations}

AQS: Annotation Quality Score; GFS: Gene function similarity; GO: Gene Ontology; PAC: Probability of Annotation Confidence

\section{Acknowledgements}

This manuscript was edited by the Office of Grants and Scientific Publications at the University of Arkansas for Medical Sciences. This work was supported by the Plant-Microbe Interfaces Scientific Focus Area in the Genomic Science Program, United States Department of Energy, Office of Science, Biological and Environmental Research. Oak Ridge National Laboratory is managed by UTBattelle, LLC, for the United States Department of Energy under Contract DEAC05-00OR22725.

\section{Funding}

No funding was obtained for this study.

\section{Availability of data and materials}

All data generated or analyzed during the current study are included in this published article and its Additional files 1 and 2.

\section{Authors' contributions}

SJ and AG conceived the project, designed the study, participated in method design, and drafted the manuscript. SJ wrote the program. IN and $\mathrm{LH}$ participated in method design, data analysis, and writing. All authors suggested ideas for additional validations that were not included into this publication, and participated in discussions. All authors read and approved the final manuscript.

\section{Ethics approval and consent to participate} Not applicable.

\section{Consent for publication}

Not applicable.

\section{Competing interests}

The authors declare that they have no competing interests.

\section{Publisher's Note}

Springer Nature remains neutral with regard to jurisdictional claims in published maps and institutional affiliations.

\section{Author details}

${ }^{1}$ Department of Biomedical Informatics, College of Medicine, University of Arkansas for Medical Sciences, Little Rock, AR 72205, USA. ${ }^{2}$ Comparative Genomics Group, Biosciences Division, Oak Ridge National Laboratory, Oak Ridge, TN 37831, USA. ${ }^{3}$ Computer Science and Mathematics Division, Oak Ridge National Laboratory, Oak Ridge, TN 37831, USA.

Received: 24 January 2017 Accepted: 13 July 2017

Published online: 19 July 2017

\section{References}

1. Kodama $Y$, Shumway M, Leinonen R. INSD. The sequence read archive: explosive growth of sequencing data. Nucleic Acids Res. 2012;40:D54-6.

2. Leggett RM, Ramirez-Gonzalez RH, Clavijo BJ, Waite D, Davey RP. Sequencing quality assessment tools to enable data-driven informatics for high throughput genomics. Front Genet. 2013;4:288.

3. Land ML, Hyatt D, Jun S-R, Kora GH, Hauser LJ, Lukjancenko O, Ussery DW. Quality scores for 32,000 genomes. Stand Genomic. 2014;9:20.

4. Médigue C, Moszer I. Annotation, comparison and databases for hundreds of bacterial genomes. Res Microbiol. 2007;158:724-36.

5. Monk JM, Charusanti P, Aziz RK, Lerman JA, Premyodhin N, Orth JD, Feist AM, Palsson BO. Genome-scale metabolic reconstructions of multiple Escherichia Coli strains highlight strain-specific adaptations to nutritional environments. Proc Natl Acad Sci U S A. 2013;110:20338-43.

6. Caspi R, Altman T, Billington R, Dreher K, Foerster H, Fulcher CA, Holland TA, Keseler IM, Kothari A, Kubo A, et al. The MetaCyc database of metabolic pathways and enzymes and the BioCyc collection of pathway/genome databases. Nucleic Acids Res. 2014;42:D459-71.

7. White $\mathrm{O}$, Kyrpides N. Meeting report. Towards a critical assessment of functional annotation experiment (CAFAE) for bacterial genome annotation. Stand Genomic. 2010;3:240-2.

8. Nelson BK. WRAPS: a system for determining the probability of prokaryotic protein annotation correctness (dissertation, University of Nebraska at Omaha, Department of Computer Science). 2013.

9. Loevenich SN, Brunner E, King NL, Deutsch EW, Stein SE, FlyBase C, Aebersold R, Hafen E, Gelbart W, Bitsoi L, et al. The Drosophila Melanogaster PeptideAtlas facilitates the use of peptide data for improved fly proteomics and genome annotation. BMC Bioinformatics. 2009;10:59.

10. Ashburner M, Ball CA, Blake JA, Botstein D, Butler H, Cherry JM, Davis AP, Dolinski K, Dwight SS, Eppig JT, et al. Gene ontology: tool for the unification of biology. The Gene Ontology Consortium Nat Genet. 2000;25:25-9.

11. Skunca N, Altenhoff A, Dessimoz C. Quality of computationally inferred gene ontology annotations. PLoS Comp Biol. 2012;8:e1002533.

12. Bell MJ, Gillespie CS, Swan D, Lord P. An approach to describing and analysing bulk biological annotation quality: a case study using UniProtKB. Bioinformatics. 2012;28:1562-8.

13. Eilbeck K, Moore B, Holt C, Yandell M. Quantitative measures for the management and comparison of annotated genomes. BMC Bioinformatics. 2009;10:67.

14. Bakke P, Carney N, Deloache W, Gearing M, Ingvorsen K, Lotz M, McNair J, Penumetcha P, Simpson S, Voss L, et al. Evaluation of three automated genome annotations for Halorhabdus utahensis. PLoS One. 2009;4:e6291.

15. Tamames J, Casari G, Ouzounis C, Valencia A. Conserved clusters of functionally related genes in two bacterial genomes. J Mol Evol. 1997:44:66-73.

16. Rogozin IB, Makarova KS, Wolf YI, Koonin EV. Computational approaches for the analysis of gene neighbourhoods in prokaryotic genomes. Brief Bioinform. 2004;5:131-49.

17. Yin $Y$, Zhang $H$, Olman $V$, Xu Y. Genomic arrangement of bacterial operons is constrained by biological pathways encoded in the genome. Proc Natl Acad Sci U S A. 2010;107:6310-5.

18. Yelton AP, Thomas BC, Simmons SL, Wilmes P, Zemla A, Thelen MP, Justice N, Banfield JF. A semi-quantitative, synteny-based method to improve functional predictions for hypothetical and poorly annotated bacterial and archaeal genes. PLoS Comput Biol. 2011;7:e1002230. 
19. UniProt C, Apweiler R, Bateman A, Martin MJ, O'Donovan C, Magrane M, Alam-Faruque Y, Alpi E, Antunes R, Arganiska J, et al. Activities at the universal protein resource (UniProt). Nucleic Acids Res. 2014;42:D191-8.

20. Tatusova T, Ciufo S, Fedorov B, O'Neill K, Tolstoy I. RefSeq microbial genomes database: new representation and annotation strategy. Nucleic Acids Res. 2014;42:D553-9.

21. Tatusov RL, Fedorova ND, Jackson JD, Jacobs AR, Kiryutin B, Koonin EV, Krylov DM, Mazumder R, Mekhedov SL, Nikolskaya AN, et al. The COG database: an updated version includes eukaryotes. BMC Bioinformatics. 2003;4:41.

22. Kanehisa M, Goto S, Sato Y, Kawashima M, Furumichi M, Tanabe M. Data information, knowledge and principle: back to metabolism in KEGG. Nucleic Acids Res. 2014;42:D199-205.

23. Finn RD, Bateman A, Clements J, Coggill P, Eberhardt RY, Eddy SR, Heger A, Hetherington K, Holm L, Mistry J, et al. Pfam: the protein families database. Nucleic Acids Res. 2014;42:D222-30.

24. Zhao S, Kumar R, Sakai A, Vetting MW, Wood BM, Brown S, Bonanno JB, Hillerich BS, Seidel RD, Babbitt PC, et al. Discovery of new enzymes and metabolic pathways by using structure and genome context. Nature. 2013; 502:698-702.

25. Xu T, Du L, Zhou Y. Evaluation of GO-based functional similarity measures using S. Cerevisiae protein interaction and expression profile data. BMC Bioinformatics. 2008;9:472.

26. The UniProt Consortium. http://www.uniprot.org. Accessed 30 May 2013.

27. The Gene Ontology Consortium. http://www.geneontology.org. Accessed 30 May 2013.

28. Dam P, Olman V, Harris K, Su Z, Xu Y. Operon prediction using both genomespecific and general genomic information. Nucleic Acids Res. 2007;35:288-98.

\section{Submit your next manuscript to BioMed Central and we will help you at every step:}

- We accept pre-submission inquiries

- Our selector tool helps you to find the most relevant journal

- We provide round the clock customer support

- Convenient online submission

- Thorough peer review

- Inclusion in PubMed and all major indexing services

- Maximum visibility for your research

Submit your manuscript at www.biomedcentral.com/submit 